\title{
The asymmetric relationship between smoking and sleep: Longitudinal findings from Dutch panel data
}

\author{
Brian M. Doornenbal ${ }^{1,2}$, Brian R. Spisak ${ }^{3,4,5}$
}

\section{AFFILIATION}

1 Department of Public Health and Primary Care, Leiden University Medical Center, Leiden, Netherlands

2 Department of Data Science, Salut., Arnhem, Netherlands

3 Department of Health Policy and Management, Harvard T.H. Chan School of Public Health, Cambridge, United States

4. Department of Data Science, HSC Analytics, Dunedin, New Zealand

5 Department of Management and Organisation, Vrije Universiteit Amsterdam, Amsterdam, Netherlands

\section{CORRESPONDENCE TO}

Brian M. Doornenbal. Data Science Department, Salut., Jansbuitensingel 7, 6811 AA, Arnhem, Netherlands. E-mail: brian@gezondmetsalut.nl ORCID ID: https://orcid.org/0000-0001-9711-4167

\section{KEYWORDS}

smoking, sleep problems, asymmetric effects, Netherlands

Received: 28 June 2021, Revised: 5 October 2021,

Accepted: 7 October 2021

\begin{abstract}
INTRODUCTION Smokers typically experience more sleep problems than non-smokers, so sleep problems are frequently reported by smokers who are attempting to quit and these can be expected to develop also after initiation of smoking. However, little is known about the effects of starting smoking on sleep problems. Some research suggests smoking reduces stress, which indicates that some aspects of smoking could benefit sleep. This study aims to contribute to the understanding of the asymmetry of the relationship between smoking and sleep problems.

METHODS To test the asymmetry of the relationship between smoking and sleep problems, a logistic regression analysis was conducted via GEE (generalized estimating equation). GEEs are an extension of Generalized Linear Models (GLMs) which allow for longitudinal data and correlation among observations of the same unit (person). This technique was recently proposed to test the asymmetry of causal effects based on panel data. The data used were from the LISS panel, a representative sample of Dutch individuals. The sample
\end{abstract}

( $n=1477)$ contained 259 individuals that changed their smoking status at least once. When conducting the analysis, we controlled for time-varying variables such as: subjective health, BMI, partner status, and parental status.

RESULTS The asymmetric effects of smoking on sleep problems were statistically significant, suggesting that starting smoking and quitting smoking are not similarly related to sleep problems. Starting smoking was not significantly related to sleep problems, but quitting smoking increased the odds of sleep problems by $23 \%$. No statistically significant relationship was found between sleeping problems and changes in BMI and parental status. Subjective health was negatively associated with sleeping problems. Stopping to live with a partner increased the likelihood of sleep problems by $41 \%$.

CONCLUSIONS Quitting smoking can have a different impact on sleep problems than starting smoking. In this study, starting smoking did not result in sleep problems, whereas quitting smoking resulted in sleep problems.

\section{INTRODUCTION}

Smoking has substantial adverse health effects, including cancer, lung diseases, and cardiovascular morbidity ${ }^{1}$. A direct impact of smoking is its effect on sleep. Smoking has been associated with an increased prevalence of sleep problems $\mathrm{s}^{2-4}$, and these sleep-related problems relate to severe health burdens associated with a number of physical and mental disorders ${ }^{4,5}$ which are costly to society ${ }^{6,7}$.

Sleep problems are not only common among smokers $2,8,9$, but are also frequently reported by smokers attempting to quit $^{3,4}$. Sleep problems experienced by quitting smokers result from nicotine craving, pharmacological agents used for smoking cessation (e.g. nicotine replacement), and weight gain 7 . Because non-smokers usually sleep better than smokers ${ }^{2,9}$, it could be presumed that starting smoking would lead to sleep problems. However, individuals often smoke to relax and relieve stress ${ }^{10}$. Some evidence suggests that smoking can alleviate stress ${ }^{11}$. Moreover, individuals who used to smoke can reduce stress by relapsing to smoking because it can alleviate withdrawal symptoms ${ }^{12}$. Overall, quitting smoking might thus result more often in sleep problems than starting smoking. 
If quitting smoking induces sleep problems and starting smoking does not reduce them, then the relationship between smoking and sleep problems is asymmetric. Theorists and data analysts usually assume that causal effects are symmetric ${ }^{13}$ - meaning that if a unit increase in variable $x$ produces a change of $b$ units in variable $y$, then a unit decrease in $x$ will result in a change of - $b$ units in $y^{14}$. However, exactly reversible causal effects are implausible for many social and psychological phenomena ${ }^{13}$. Whether a relationship is symmetric is an empirical question for which good methodological tools have been lacking ${ }^{14}$. Recently, Allison ${ }^{14}$ proposed a technique to test the asymmetry of causal effects based on panel data. It is thus possible to test whether the effects of smoking on sleep are indeed asymmetric. This study, accordingly, examines the asymmetry of the relationship between smoking and sleep problems by applying techniques proposed by Allison ${ }^{14}$.

Testing the asymmetry of the relationship between smoking and sleep problems is important because it increases the understanding of the dangers of smoking to sleep. If individuals who start smoking do not experience sleep problems directly, then they may feel less discouraged from smoking. Smoking might not cause (or even help to reduce) sleep problems when starting but results in sleep problems over time. A better understanding of the asymmetry of the effects of smoking on sleep is therefore also of practical importance for awareness programs about the hidden dangers of tobacco.

\section{METHODS}

\section{Data}

The data used for testing the asymmetry of the effects of smoking on sleep are from the LISS (Longitudinal Internet Studies for the Social sciences) panel, a representative sample of Dutch individuals ${ }^{15}$. The LISS survey is administrated annually by CentERdata (Tilburg University, The Netherlands) and consists of questions on themes such as personality, family, and health. The LISS data are freely available for academic research on the website www.lissdata.nl. More information about the LISS panel, including how researchers can use the LISS data is described in Scherpenzeel ${ }^{15}$.

\section{Variables}

\section{Smoking measure}

The independent variable (smoking) was measured through the question: 'Do you smoke now?'. With response option 'yes' or 'no'. Based on the answer to that question over two consecutive years, change of smoking status was inferred. Participants started smoking $(+1)$ when their answer was 'yes' in the current year and 'no' in the previous year, while participants quitted smoking (-1) when their answer was 'no' in the current year, but 'yes' in the previous year. Change in smoking status was treated as a zero (0) when participants did not change their smoking status. Finally, it is important to note that although people tend to underestimate how much they smoke ${ }^{16}$, self-reports of smoking have been found to be accurate in most studies ${ }^{17}$.

\section{Sleep measure}

Overall sleep problems were measured by asking the participants directly: 'Do you regularly suffer from sleeping problems?'. During the analysis, the response from the previous year was compared with the current response. A change from 'no' to 'yes' was treated as an increase in sleeping problems (+1), and a 'yes' to 'no' was treated as a decrease in sleeping problems $(-1)$.

\section{Control variables}

While testing the asymmetry of the effects of smoking on sleep over time, the analytical approach only allowed for timevarying variables to be included as main effects ${ }^{18,19}$. Hence, variables such as gender and psychiatric history status (e.g. depression, anxiety) were not included in the model - noting that we capture fluctuations in subjective health.

Besides the onset and offset of smoking, we were able to control for changes in the following time-varying variables previously found to affect sleep problems: subjective health, BMI, partner status, and parental status. Subjective health was measured using the item: 'How would you describe your health, generally speaking?' (1='poor' to 5='excellent'); BMI was computed $\left(\mathrm{kg} / \mathrm{m}^{2}\right)$; partner status assessed by the two questions 'Do you currently have a partner?' and 'Do you live together with this partner?' (1=living with a partner, $0=$ not living with a partner); and parental status by 'Did you ever have any children?' (1='yes', $0=$ 'no').

\section{Statistical analysis}

To test the asymmetry of the causal effects ${ }^{14}$ of smoking on sleep problems, a logistic regression analysis was conducted via generalized estimating equation (GEE). GEEs are an extension of Generalized Linear Models (GLMs) which allow for longitudinal data and correlation among observations of the same unit (person). Rather than comparing smokers with non-smokers, as in conventional cross-sectional regression models, only within-person variation was used to estimate the parameters. The within-person variation in predictor variables (including control variables) were decomposed into positive (e.g. starting smoking) and negative variables (e.g. quitting smoking). For example, when a person started smoking, the positive variable of smoking was coded as 1 for that person (otherwise 0 ). When a person quit smoking, the negative variable of smoking was coded as 1 for that person (otherwise 0). Measures on an ordinal (i.e. subjective health) and continuous (i.e. BMI) scale were coded as the absolute increase and decrease. By focusing on withinperson variation, the procedure is suitable for testing the consequences of increases and decreases in a variable of interest ${ }^{14}$. The regression model controlled for time-invariant heterogeneity by removing between-individual variance, resulting in each individual serving as his/her own control ${ }^{14}$. 
The asymmetry of the effects was tested by comparing the relationship between the positive variables (e.g. starting smoking) and sleep problems with the relationship between the negative variables (e.g. quitting smoking) and sleep problems (chi-squared test).

\section{RESULTS}

The longitudinal panel data used in this study were fielded during five waves, in 2015, 2016, 2017, 2018, and 2019. During these waves, 1477 unique individuals completed the full questionnaire. Completed data were necessary to compute the asymmetry of the effects for fixed time intervals. The sample used in the analysis included 259 unique individuals $(17.5 \%$; 1295 records in total) who changed their smoking status at least once and at most four times (mean=1.4, $\mathrm{SD}=0.6$ ). The majority of these individuals (67\%) changed their smoking status only once. About half of the participants that changed their smoking status at least once (52\%) were women aged $18-87$ years, mostly within a bandwidth 40.6-70.4 years (mean $=55.5, \mathrm{SD}=14.9$ ). Most of these participants were part of a household of two members (mean=2.2, $\mathrm{SD}=1.0)$ and had a partner $(65 \%)$ and/ or a child (70\%). The average BMI was $26.8 \mathrm{~kg} / \mathrm{m}^{2}$ (SD=11.2). Table 1 provides an overview of the participants, including comparisons between the individuals that changed their smoking status $(n=259)$ and the individuals that did not change their smoking status $(\mathrm{n}=1218)$.

The results of the logistic regression analysis are presented in Table 2. A one-step improvement of subjective health reduced the odds of sleep problems by $21 \%$ (B=-

Table 1. Characteristics of participants included in the study

\begin{tabular}{|c|c|c|c|c|c|c|}
\hline \multirow[t]{2}{*}{ Characteristics } & \multicolumn{2}{|c|}{$\begin{array}{l}\text { Change in smoking status } \\
(\mathrm{n}=259)\end{array}$} & \multicolumn{2}{|c|}{$\begin{array}{l}\text { No change in smoking status } \\
(\mathrm{n}=1218)\end{array}$} & \multicolumn{2}{|c|}{$\begin{array}{c}\text { Total } \\
(\mathrm{n}=1477)\end{array}$} \\
\hline & Mean (\%) & SD & Mean (\%) & SD & Mean (\%) & SD \\
\hline Female $^{a}$ & 52 & - & 44 & - & 46 & - \\
\hline Age (years) $^{\mathrm{b}}$ & 55.5 & 14.9 & 64.6 & 12.5 & 63.2 & 13.3 \\
\hline Partner status ${ }^{c}$ & 65 & - & 73 & - & 71 & - \\
\hline Parental status ${ }^{\mathrm{d}}$ & 70 & - & 81 & - & 79 & - \\
\hline BMI $\left(\mathrm{kg} / \mathrm{m}^{2}\right)^{\mathrm{e}}$ & 26.8 & 11.2 & 27.8 & 50.5 & 27.67 & 46.1 \\
\hline
\end{tabular}

Partner status and parental status indicate whether the participants have a partner and a child. Student's t-test was used to test the mean difference between the participants that changed smoking status and participants that did not change smoking status. a The group that changed their smoking status had a higher proportion of woman than the group that did not change their smoking status, $\mathrm{t}(1475)=2.2, \mathrm{p}<0.05$. $\mathrm{b}$ The group that changed their smoking status were younger than the group that did not change their smoking status, $\mathrm{t}(1475)=9.1, \mathrm{p}<0.001$. $\mathrm{c}$ Individuals from the group that changed their smoking status had a partner less often than individuals from the group that did not change their smoking status, $t(1475)=2.6, p<0.01$. d Individuals from the group that changed their smoking status were a parent less often than individuals from the group that did not change their smoking status, $\mathrm{t}(1475)=4.0, \mathrm{p}<0.001$. e The BMI of individuals from the group that changed their smoking status was not significantly different from the BMI of individuals from the group that did not change their smoking status, $\mathrm{t}(1475)=0.3$, non-significant.

Table 2. Results of asymmetric logistic regression via GEE, estimating changes in sleep problems

\begin{tabular}{lcccc} 
Parameter & $\mathbf{B}(95 \% \mathbf{C I})$ & SE & $\mathbf{z}$ & $\mathbf{p}$ \\
\hline Intercept & $-1.18(-1.30--1.06)$ & 0.06 & -18.70 & $<0.001$ \\
Subjective health (+) & $-0.21(-0.32--0.09)$ & 0.06 & -3.54 & $<0.001$ \\
Subjective health (-) & $0.18(0.08-0.28)$ & 0.05 & 3.53 & $<0.001$ \\
BMI (+) & $0.00(0.00-0.00)$ & 0.00 & -1.97 & 0.049 \\
BMI (-) & $0.00(-0.00-0.00)$ & 0.00 & 0.42 & 0.677 \\
Partner status (+) & $0.38(-0.10-0.85)$ & 0.24 & 1.56 & 0.119 \\
Partner status (-) & $0.41(0.01-0.81)$ & 0.20 & 1.99 & 0.046 \\
Parental status (+) & $-0.34(-0.85-0.18)$ & 0.26 & -1.28 & 0.199 \\
Parental status (-) & $-0.14(-0.51-0.23)$ & 0.19 & -0.75 & 0.451 \\
Smoking (+) & $0.18(-0.09-0.45)$ & 0.14 & 1.32 & 0.188 \\
Smoking (-) & $0.23(0.01-0.44)$ & 0.11 & 2.07 & 0.039 \\
t2 (wave 2017) & $0.00(-0.00-0.00)$ & 0.04 & -0.01 & 0.990 \\
t3 (wave 2018) & $0.00(-0.00-0.00)$ & 0.05 & 0.08 & 0.938 \\
t4 (wave 2019) & $0.00(-0.00-0.00)$ & 0.06 & -0.04 & 0.970
\end{tabular}

The onset of smoking (and other variables) is (are) denoted by (+). The offset of smoking (and other variables) is (are) denoted by (-). 
$0.21, \mathrm{p}<0.001$ ) and a one-step deterioration of subjective health increased the odds by $18 \%(\mathrm{~B}=0.18, \mathrm{p}<0.001)$. Partner status was also significantly associated with sleep problems. Starting to live together with a partner had no significant impact on sleep problems $(B=0.38, p=0.12)$, but stopping living together increased the likelihood of sleep problems by $41 \%(B=0.41, \mathrm{p}<0.05)$. The asymmetric effects of partner status on sleep problems were statistically significant $\left(\chi^{2}=6.4, p=0.01\right)$. Finally, starting smoking did not significantly increase the odds of sleep problems $(B=0.18, p=0.19)$. In contrast, quitting smoking increased the likelihood of sleep problems by $23 \%(B=0.23, p<0.05)$. The asymmetric effects of smoking on sleep problems were significant $\left(\chi^{2}=6.0, p=0.01\right)$, indicating that starting smoking and quitting smoking did not have opposite effects on sleep problems. Thus, quitting smoking is indeed followed by sleep problems, whereas starting smoking is not significantly related to sleep problems.

\section{DISCUSSION}

The aim of this study was to test the asymmetry of the relationship between smoking and sleep problems. Based on Dutch panel data, the analysis revealed that starting smoking does not have a similar relationship with sleep problems as quitting smoking. Quitting smoking was associated with an increase in sleep problems, whereas starting smoking was not significantly related to sleep problems. Smoking might indeed not cause sleep problems when starting smoking but could result in sleep problems over time.

The findings of this study are in line with previous research pointing out that smokers attempting to quit encounter sleep problems ${ }^{3,4}$. It also expands the understanding of the impact of starting smoking on sleep problems. Previous research suggests that smoking reduces stress ${ }^{11}$, which indicates that some aspects of smoking may benefit sleep. Furthermore, for people who used to smoke, starting smoking can help alleviate sleep-disrupting withdrawal symptoms ${ }^{2,9}$. However, as previous research also suggests that smokers sleep worse than non-smokers, it is reasonable to assume that starting smoking eventually causes sleep problems. Indeed, based on the current data, the asymmetrical effects found do not support a reduction in sleep problems as a result of smoking. Although the direction of the impact of smoking on sleep problems was positive, the effect of starting smoking was not significant.

The lack of support for a positive impact on sleep problems raises the question when smokers start to experience more sleep problems than non-smokers. Possibly, sleep problems may have already increased before persons start smoking, as smoking could be perceived as a way to relax and relieve stress ${ }^{10}$. Starting smoking, similarly, can alleviate withdrawal symptoms for individuals that used to smoke 4 . However, it should be noted that in the present sample, the majority of participants only changed their smoking status once. Hence, our findings mostly apply to individuals who started or stopped smoking once during the five measurements (i.e. a five-year period ${ }^{a}$ )

In short, future research should consider testing whether existing sleep problems based on psychological (e.g. stress) and physiological factors (e.g. withdrawal) lead individuals to start smoking.

\section{Limitations}

While further examining the relationship between smoking and sleep problems, two important study limitations should be considered. First, although we controlled for several important factors affecting sleep, the relationship between smoking and sleep problems can be affected by factors such as smoking history, heaviness of smoking, and the use of replacement therapy. Future research should test how these factors moderate the (asymmetry of the) relationship between smoking and sleep problems. Possibly, individuals who become heavier first-time smokers experience more sleep issues as well as when they begin smoking. Second, the validity of our measurements warrant consideration. Because the measurements were applied only once each year, within-year changes were not accounted for. As a result, our measurements are sensitive to misclassification bias. For example, if participants started smoking just after their last measurement, any sleep problems arising from starting smoking might have ended by the time of the next measurement. Likewise, our measurement of sleep disturbance is brief and easy to administer, but uncommon in the sleep literature and perhaps less valid. More established measures of sleep problems are the Pittsburgh Sleep Quality Index (PSQI) ${ }^{20}$, Jenkins Sleep Scale (JSS) ${ }^{21}$, and the General Sleep Disturbance Scale (GSDS) ${ }^{22}$. Although our measurement (i.e. 'Do you regularly suffer from sleeping problems?') is closely related to the one-item sleep scale of the quality of life questionnaire (EORTC QLQ-C30: 'Have you had trouble sleeping?'), which is found to be related to the Jenkins Sleep Scale $^{23}$. Future research should test whether our findings hold while using more established measures.

\section{CONCLUSIONS}

The relationship between quitting smoking and sleep problems can be dissimilar from the relationship between starting smoking and sleep problems. Based on longitudinal panel data, this study found an increase in sleep problems after quitting smoking, but no statistically significant change in sleep problems after starting smoking. Awareness programs focused on the dangers of smoking should consider pointing out these delayed, hidden dangers of smoking.

\section{REFERENCES}

1. Chang JT, Anic GM, Rostron BL, Tanwar M, Chang CM. Cigarette Smoking Reduction and Health Risks: A Systematic Review and Meta-analysis. Nicotine Tob Res. 2021;23(4):635-642. doi:10.1093/ntr/ntaa156

a A post hoc analysis of a sub-sample of those who quit and relapsed ( $\mathrm{n}=56$ ) did not provide support for a significant (or asymmetric) relationship between smoking and sleep problems. 
2. Cohrs S, Rodenbeck A, Riemann D, et al. Impaired sleep quality and sleep duration in smokers-results from the German Multicenter Study on Nicotine Dependence. Addict Biol. 2014;19(3):486-496. doi:10.1111/j.1369-1600.2012.00487.x

3. Colrain IM, Trinder J, Swan GE. The impact of smoking cessation on objective and subjective markers of sleep: Review, synthesis, and recommendations. Nicotine Tob Res. 2004;6(6):913-925. doi:10.1080/14622200412331324938

4. Jaehne A, Loessl B, Bárkai Z, Riemann D, Hornyak M. Effects of nicotine on sleep during consumption, withdrawal and replacement therapy. Sleep Med Rev. 2009;13(5):363-377. doi:10.1016/j.smrv.2008.12.003

5. Irwin MR, Olmstead R, Carroll JE. Sleep Disturbance, Sleep Duration, and Inflammation: A Systematic Review and Meta-Analysis of Cohort Studies and Experimental Sleep Deprivation. Biol Psychiatry. 2016;80(1):40-52. doi:10.1016/j.biopsych.2015.05.014

6. Hillman D, Mitchell S, Streatfeild J, Burns C, Bruck D, Pezzullo L. The economic cost of inadequate sleep. Sleep. 2018;41(8):10.1093/sleep/zsy083. doi:10.1093/sleep/zsy083

7. Htoo A, Talwar A, Feinsilver SH, Greenberg H. Smoking and sleep disorders. Med Clin North Am. 2004;88(6):1575-1591. doi:10.1016/j.mcna.2004.07.003

8. Costa M, Esteves M. Cigarette Smoking and Sleep Disturbance. Addict Disord Their Treat. 2018;17(1):40-48. doi:10.1097/ADT.0000000000000123

9. Sujarwoto S. Sleep Disturbance in Indonesia: How Much Does Smoking Contribute? Behav Sleep Med. 2020;18(6):760-773. doi:10.1080/15402002.2019.1682584

10. Stubbs B, Veronese N, Vancampfort D, et al. Perceived stress and smoking across 41 countries: A global perspective across Europe, Africa, Asia and the Americas. Sci Rep. 2017;7(1):7597. doi:10.1038/s41598-017-07579-w

11. Choi D, Ota S, Watanuki S. Does cigarette smoking relieve stress? Evidence from the event-related potential (ERP). Int J Psychophysiol. 2015;98(3)(Pt 1):470-476. doi:10.1016/j.ijpsycho.2015.10.005

12. Patterson F, Grandner MA, Malone SK, Rizzo A, Davey A, Edwards DG. Sleep as a Target for Optimized Response to Smoking Cessation Treatment. Nicotine Tob Res. 2019;21(2):139-148. doi:10.1093/ntr/ntx236

13. Lieberson S. Making It Count: The Improvement of Social Research and Theory. University of California Press; 1987.

\section{CONFLICTS OF INTEREST}

The authors have each completed and submitted an ICMJE Form for Disclosure of Potential Conflicts of Interest. The authors declare that they have no competing interests, financial or otherwise, related to the current work. B. M. Doornenbal reports funds from Leiden University Medical Center and Salut., and honoraria from Erasmus University Rotterdam, outside the submitted work.

FUNDING

There was no source of funding for this research.

ETHICAL APPROVAL AND INFORMED CONSENT

Ethical approval and informed consent were not required for this study,

\section{doi:10.1525/9780520908420}

14. Allison PD. Asymmetric Fixed-effects Models for Panel Data. Socius. 2019;5:1-12. doi:10.1177/2378023119826441

15. Scherpenzeel A. Data Collection in a Probability-Based Internet Panel: How the LISS Panel Was Built and How It Can Be Used. Bull Methodol Sociol. 2011;109(1):56-61. doi:10.1177/0759106310387713

16. Connor Gorber S, Schofield-Hurwitz S, Hardt J, Levasseur G, Tremblay M. The accuracy of self-reported smoking: A systematic review of the relationship between self-reported and cotinine-assessed smoking status. Nicotine Tob Res. 2009;11(1):12-24. doi:10.1093/ntr/ntn010

17. Patrick DL, Cheadle A, Thompson DC, Diehr P, Koepsell T, Kinne S. The validity of self-reported smoking: a review and meta-analysis. Am J Public Health. 1994;84(7):1086-1093. doi:10.2105/ajph.84.7.1086

18. Gangl M. Causal Inference in Sociological Research. Annu Rev Sociol. 2010;36:21-47. doi:10.1146/annurev.soc.012809.102702

19. Gunasekara FI, Richardson K, Carter K, Blakely T. Fixed effects analysis of repeated measures data. Int J Epidemiol. 2014;43(1):264-269. doi:10.1093/ije/dyt221

20. Buysse DJ, Reynolds CF, Monk TH, Berman SR, Kupfer DJ. The Pittsburgh sleep quality index: A new instrument for psychiatric practice and research. Psychiatry Res. 1989;28(2):193-213. doi:10.1016/0165-1781(89)90047-4

21. Jenkins CD, Stanton BA, Niemcryk SJ, Rose RM. A scale for the estimation of sleep problems in clinical research. J Clin Epidemiol. 1988;41(4):313-321. doi:10.1016/0895-4356(88)90138-2

22. Lee KA. Self-reported sleep disturbances in employed women. Sleep. 1992;15(6):493-498. doi:10.1093/sleep/15.6.493

23. Hofmeister D, Schulte T, Hinz A. Sleep problems in cancer patients: a comparison between the Jenkins Sleep Scale and the single-item sleep scale of the EORTC QLQ-C30. Sleep Med. 2020;71:59-65. doi:10.1016/j.sleep.2019.12.033

as existing data from the Longitudinal Internet studies for the Social Sciences (LISS) panel were used. The LISS survey is administrated annually by CentERdata (Tilburg University, The Netherlands). Further information about ethical approval of LISS can be found at www.lissdata. nl/faq-page\#n5512.

\section{DATA AVAILABILITY}

The data supporting this research are available from the following source: www.lissdata.nl

\section{PROVENANCE AND PEER REVIEW}

Not commissioned; externally peer reviewed. 